\title{
HISTORIA SOCIAL DEL DERECHO PARA Y DESDE AMÉRICA LATINA
}

\section{Daniel Sandoval}

\section{Cervantes ${ }^{1}$}

\section{Resumen}

El objetivo del escrito es proponer la incorporación de los conceptos de la historia social para la construcción de una historia crítica del derecho en América Latina. Primero se establece qué se entiende por derecho e historia, después las posibilidades que la historia social puede traer a la explicación del derecho; en seguida se analiza el tiempo histórico, sobre todo, la larga duración en relación con el derecho, por último unas breves notas para la construcción de una historia social del derecho en América Latina. Los resultados son aún preliminares, pues la investigación se encuentra en sus primeras etapas.

Palabras clave: Derecho; Historia; Derechos sociales y económicos; América Latina; México.

\section{INTRODUCCIÓN}

El presente trabajo constituye un primer esbozo del papel que la historia social y la historia crítica pueden tener en la explicación del papel real del derecho en el desarrollo del capitalismo y en la conformación de las relaciones sociales dominantes, pensando específicamente en México y en América Latina. Recuperar un análisis histórico-crítico sobre dicho tema es importante por dos razones: la primera, la casi inexistente literatura que aborde el derecho en América Latina desde una perspectiva crítica e interdisciplinaria como es la propuesta de la historia social; la segunda, la necesidad de encontrar explicaciones adecuadas sobre el pasado del derecho en nuestra

\footnotetext{
${ }^{1}$ Profesor-investigador Titular B, Tiempo Completo. Departamente de Ciencias Jurídicas, Instituto de Ciencias Sociales y Administrativas, Universidad Autónoma de Ciudad Juárez. Becario del Programa de Becas Posdoctorales de la UNAM, Centro de Investigaciones Interdisciplinarias en Ciencias y Humanidades, UNAM. E-mail: dscervantes@hotmail.com
} 
región para estar en condiciones de entender el presente y, a partir de ahí, construir o fortalecer mejores opciones para el futuro.

El trabajo parte de dos enfoques metodológicos principales: aquél aportado por la Crítica Jurídica, principalmente por Oscar Correas, del cual se toman las categorías pertinentes (como la de sentido ideológico del derecho, o la distinción entre discurso del derecho y discurso jurídico) para analizar desde una perspectiva jurídica crítica, pero abierta a enfoques provenientes de otros campos disciplinares del saber; así como también varias vertientes de la tradición marxista de la historia social, aparato crítico que nos permitirá percibir el fenómeno jurídico como uno colocado en el tiempo y en el espacio y en contacto con lo político, lo social, lo cultural y lo económico. De manera que ambas perspectivas se complementan para comprender lo jurídico dentro de la totalidad que es la vida social.

Para realizar el objeto principal, el escrito se dividirá en cuatro partes: en la primera se asentará tanto el concepto de derecho como el de historia desde los cuales se parte; en la segunda, se abordará la manera en que desde dichas concepciones se puede comprender a lo jurídico desde una perspectiva de totalidad; en la tercera parte se planteará la necesidad de repensar el derecho desde un enfoque histórico crítico tanto para poder explicar cuáles han sido las condiciones de emergencia y de funcionamiento del derecho en el pasado como también, a partir de ello, comprender cuáles son éstas en el futuro y cómo pueden transformarse en el futuro; en la cuarta sección se estudiará la importancia de entender lo jurídico en la larga duración y de comprender los diferentes tiempos históricos que deben ser tomados en cuenta para analizar al derecho.

\section{¿QUÉ DERECHO? ¿QUÉ HISTORIA?}

La primera pregunta que surge en el camino de una explicación histórica-crítica del derecho es, precisamente, ¿qué se entiende por el concepto mismo de derecho? La pregunta es importante, pues, lo que se entiende por "derecho" es producto de la manera, de las categorías y la metodología, desde la cual se aborda el tema, la cual enmarca ya, aún de manera muy general, las posibilidades de explicación del fenómeno. En este sentido, parto del concepto de derecho que ha establecido la crítica jurídica, particularmente Oscar Correas, pues pensamos que es a partir de éste que es posible 
explicar desde una perspectiva crítica y tendencialmente interdisciplinaria el campo de lo jurídico.

Hay que comenzar por entender al derecho como un discurso. Aclarando dos cuestiones: la primera es que se opta por considerarlo como un discurso, entendiendo que la característica primordial que distingue algo reconocido como derecho de otros fenómenos que no son considerados como jurídicos es su carácter discursivo, es decir, que se ejerce a través de un discurso reconocido como derecho, tanto en sus condiciones de construcción como en las de su ejercicio. La segunda cuestión es que percibir al derecho como un discurso, no implica, en lo absoluto, desconocerlo como una práctica social, es decir, no implica invisibilizar su construcción social y los efectos de su aplicación en lo social, al contrario, intenta visibilizar éstos tratando de comprender la especificidad de lo jurídico desde una perspectiva de la totalidad social (CORREAS, 2005: 27-64).

En este sentido, el derecho es considerado como un discurso prescriptivo que amenaza con el ejercicio de la violencia física. Esta definición, si bien no es propia de la crítica jurídica, si ha sido explicada de una manera específica por ésta (KELSEN, 2007: 44-58; WEBER, 2002: 1056-106o; CORREAS, 2005: 65-95). En primer lugar, puesto que, si el derecho es un discurso, una parte importante de comprenderlo proviene del sentido que se le reconozca a éste. La crítica jurídica ha puesto especial énfasis en que el sentido del discurso del derecho es producto de la lucha de clase y de las maneras en que se resuelven, siempre de manera momentánea, las relaciones de fuerzas de nuestras sociedades dividas en clases.

De esta manera podemos observar la primera innovación de la concepción crítica jurídica del derecho: sus contenidos, dentro de los cuales se encuentran las normas jurídicas, son producto de relaciones sociales y de conflictos de clase concretos. El derecho es siempre un resultado de la sociedad desde la cual se produce, a la vez que tiene efectos sobre su reproducción (CORREAS, 2004: 24; WOLKMER, 2012: 45-55).

En segundo lugar, entender la derecho como discurso, desde la crítica jurídica, implica entenderlo como no solamente formado por normas jurídicas, sino también como conteniendo algo más. Aquí la distinción entre discurso derecho, el cual se define como aquel que relaciona un modalizador deóntico (obligatorio, prohibido, permitido) a una conducta de forma prescriptiva, y discurso jurídico, entendido como cualquier 
discurso que se refiera al anterior, resulta importante, pues, a partir de ésta se puede explicar el papel que la ideología -entendida en general como contenidos de conciencia - tiene en la producción del discurso del derecho propiamente dicho, es decir, sobre las normas jurídicas (CORREAS, 2005, p. 4).

Por otro lado, el papel de la ideología en el discurso del derecho no termina ahí, sino que se vehiculiza a través del sentimiento de deber que despierta en la conciencia de los ciudadanos el discurso del derecho (CORREAS: 159-161). Para ello es necesario agregar la distinción entre sentido deóntico y sentido ideológico del discurso del derecho, esencial para comprender la manera en que la ideología dominante también le imprime su sentido, no solamente al discurso jurídico, sino, principalmente, al discurso del derecho, a aquél que, en parte, se forma por las normas jurídicas. De manera que se pueda explicar el papel que tiene el derecho, en su conjunto, en la reproducción del régimen capitalista, dominante y en vías constantes de construcción de hegemonía en la mayor parte de las civilizaciones contemporáneas.

En este sentido, si bien las normas jurídicas, generalmente, son explicadas desde su sentido deóntico, aquel que regula prescriptivamente las conductas, también puede ser entendidas desde su sentido ideológico, cuyo efecto es la construcción de la conciencia del dominado en los ciudadanos, la cual se puede intentar definir como aquel estado de conciencia que naturaliza lo ordenado por el sentido deóntico de las normas jurídicas como moralmente bueno, es decir, como algo moralmente debido (CORREAS, 2005: 147-149).

Por ejemplo, el sentido deóntico de las normas jurídicas relativas a la regulación de las relaciones laborales impone, jurídicamente, un conjunto de obligaciones tanto a los trabajadores como a los patrones. Por medio del sentido ideológico, contenido por ejemplo, en la definición del trabajo como el intercambio equivalente entre el salario, entregado por el capitalista al trabajador, y el trabajo, entregado por el trabajador al capitalista. Definición detrás de la cual se esconde todo el conjunto de mecanismos de extracción de plusvalor, que implican pagar al trabajador un monto menor al que produjo, implican que el salario solamente es posible en cuanto una parte de la fuerza 
del trabajador se aleja de él en forma de plusvalor (MARX, 1999: 477-478). ${ }^{2}$ De manera que el sentido ideológico del derecho implica la naturalización de un conjunto de relaciones sociales inequitativas y necesarias para la reproducción del capital, a través de la naturalización de las normas jurídicas que las regulan y las ayudan a reproducirse.

Por último, en cuanto a la relación entre la violencia y el derecho, la crítica jurídica concibe al derecho no como un simple medio de ejercer la violencia socialmente legítima, sino como el discurso que la organiza y la hace socialmente posible, es decir, la amenaza de la violencia no ocupa una posición extrínseca de garantía última de cumplimiento (HART, 1998: 129-135). Sino, al contrario, la cualidad de organizador de la violencia es una característica constitutiva del derecho (BENJAMIN, 2007: 113-138). ${ }^{3}$

Ahora bien, estas definiciones que tomamos de la crítica jurídica nos permiten analizar el derecho desde una perspectiva distinta a la dominante y, sin embargo, por sí solas no son suficientes para analizar su papel en la producción y reproducción de lo social en una civilización específica. Para ello se requiere del auxilio y complemento de los aportes realizados desde otros campos del saber. De ahí el acercamiento de la crítica jurídica a diversas disciplinas, como la sociología, la ciencia política, la economía, entre otras. En nuestro caso nos aproximaremos a la historia, específicamente, a la historia social, tanto porque ha sido una de las conexiones menos avanzadas desde la crítica jurídica (la cual se ha concentrado en sus relaciones tanto a la economía política como a la sociología crítica), como también porque la historia social puede contribuir de manera importante en la explicación del derecho desde una perspectiva materialista y desde la totalidad de los social.

De esta forma partiremos de pensar la historia como aquella actividad de dedicada a explicar la vida de las civilizaciones en el tiempo, principalmente el pasado (BLOCH, 2001, p. 139; BRAUDEL, 1999: 24-25). Esta definición es simple y más un punto

\footnotetext{
2 "Si el fondo de trabajo afluye a él constantemente en forma de medios de pago de su trabajo es, sencillamente, porque su propio producto se aleja de él en forma de capital. Pero esta forma de manifestarse el fondo de trabajo no altera para nada el hecho de que el capitalista desembolsa, para pagar al obrero, el propio trabajo materializado de éste", (MARX, 1999, pp. 477-478).Karl Marx, El capital. Crítica a la economía política. Tomo I, (México: Fondo de Cultura Económica, 1999), 477478.

${ }^{3}$ Parecida a la forma en que Walter Benjamin también lo observa, si bien desde la distinción entre violencia constitutiva y constituida, véase Walter Benjamin, "Para una crítica de la violencia", en Conceptos de Filosofía de la Historia, Walter Benjamin (La Plata: Terramar, 2007), 113-138.
} 
de partida que uno de llegada. Habría que destacar y explicar algunos puntos del por qué elegimos esta definición para clarificar el concepto de historia que pensamos es el más acertado para el presente trabajo y para la investigación que espero llevar a cabo a partir de éste.

En primer término, pensar la historia como un análisis de las civilizaciones tiene el propósito de destacar su pretensión de estudiar la totalidad de la vida social, por tanto, de abordar la explicación de las civilizaciones como totalidades. De aquí se puede percibir el carácter tendencialmente interdisciplinario de la historia, específicamente de la historia social, la cual busca incorporar a sus aportaciones todas aquéllas destinadas a explicar algún campo de la vida social, como son la sociología, la economía, la demografía, entre otras (BRAUDEL, 1999: 37-40). En nuestro caso, se trataría de incorporar las explicaciones en torno al papel del derecho en la formación y reproducción, o ruptura de las civilizaciones, específicamente de las sociedades latinoamericanas modernas.

Por otro lado, señalar que es análisis de las civilizaciones en el tiempo tiene dos razones: la primera, es no limitar las posibilidades de explicación de la historia al pasado solamente, pues, si bien es su objetivo principal, lo cierto es que, muchas veces, la comprensión del pasado es necesaria para poder entender el presente y, viceversa, también el entendimiento del presente es importante para analizar el pasado de una manera adecuada. De manera que la historia no es una disciplina que se limite a estudiar el pasado (BLOCH, 2001, p. 148; BRAUDEL, 1999, p. 82). La segunda razón, quizá un poco más escondida, tiene que ver con la necesidad de hacer ver que la historia tendrá que explicar tiempos con diferentes racionalidades, no solamente tiene que enfrentarse con el tiempo biológico, medido en segundos, días, años o siglos, sino con temporalidades vinculadas al contexto socio-político del cual emergen (BLOCH, 2001: 140-141; KOSELLECK, 1993: 14-15). Estas dos características serán analizadas con más profundidad en los siguientes apartados del trabajo.

Por último, antes de pasar al cuerpo del escrito propiamente dicho, me gustaría hacer algunas aclaraciones en torno al concepto de racionalidad y al de proceso histórico que me parecen relevantes. Por un lado, la historia social, y en general la historia crítica, replantea la necesidad de explicar racionalmente los procesos históricos, de entender de manera coherente y sistemática las los factores que son las condiciones 
necesarias para la realización de un hecho histórico (HOBSBAWM, 1998: 73-78; THOMPSON, 2001: 446-458; VILAR, 1978: 149-152). Esta necesidad de una explicación racional no es un obstáculo para realizar uno de los objetivos más importantes para la crítica jurídica: poner en cuestión la forma ostensiva en que se ha nombrado lo que es derecho y lo que no es derecho, partiendo de una racionalidad, la moderno-capitalista, y excluyendo otras formas de racionalidad, como las originarias (CORREAS 2004: 28-29; CORREAS 2005: 113-114).

Lo anterior debido a que, en ambos casos estamos tratando de conceptos distintos de racionalidad, sin que uno excluya necesariamente el otro. Por un lado, la racionalidad histórica tiene como objetivo explicar, de la manera más rigurosa posible, las condiciones de causación de los hechos históricos, en este sentido, la palabra racionalidad se refiere precisamente a tomar en consideración, de la manera más completa posible, todos los factores socio-político-económicos que contribuyeron -sin que uno de ellos sea la causa determinante- para la ocurrencia de los hechos históricos, su intención es explicar de manera ordenada los hechos históricos, pensándolos dentro de un proceso más general, pero articulado desde los hechos específicos y concretos (THOMPSON, 2001: 450-455; VILAR, 1998: 93; BENOIT, 1978: 14).

Por el contrario, la racionalidad jurídica cuya crítica hacemos se refiere a ese conjunto de principios que ordenan la forma de concebir, de producir y de aplicar el derecho en las sociedades modernas-capitalistas que se construyó, precisamente, de manera ahistórico, es decir, prescindiendo de dar cuenta de las condiciones y de los factores sociales, políticos y económicos que, en conjunto, determinaron su emergencia y desarrollo. En este sentido, criticar la racionalidad jurídica capitalista es, precisamente, criticar la ideología jurídica que se construye a sí misma como racionalidad a partir de la abstracción. De manera que el concepto de racionalidad y de necesidad utilizado por diversos historiadores sociales, no solamente no es contrario a este objetivo desmitificante de la crítica jurídica, sino que tiene, en cuanto se observa a la explicación histórica como contraria las mitificaciones ideológicas, principalmente nacionalistas, una herramienta complementaria (HOBSBAWM, 1996: 87-89). 


\section{TOTALIDAD, DERECHO Y CAPITALISMO. LAS POSIBILIDADES DE EXPLICACIÓN DEL DERECHO MODERNO DESDE LA HISTORIA SOCIAL}

El derecho moderno-capitalista es uno de los instrumentos de poder del régimen capitalista (CORREAS, 2004: 146-148). Esta afirmación, por un lado, implica una relación instrumental entre este derecho, históricamente localizable, y el régimen capitalista; por el otro, al ser solamente uno de los instrumentos, implica que el derecho es uno de los circuitos de poder a través de los cuales se construye y se reproduce el régimen capitalista, mas no el único. Por tanto, lo anterior quiere decir que el derecho es sólo una pieza dentro de éste.

En cuanto a la relación instrumental entre el derecho moderno y el régimen capitalista, habría que matizarla, no constituye una relación lineal y unilateral, pues, el derecho para presentarse como tendencialmente universal requiere de ser expresado en un lenguaje abstracto y que permita contener las contradicciones de clase necesarias para que pueda interpelar no solamente a la clase dominante, y todas sus facciones, sino también a las clases subalternas (MARX \& ENGELS, 1974: 71-72). ${ }^{4}$ Buena parte del éxito del derecho moderno-capitalista como instrumento de poder consiste en que se consolide como una forma de resolución de conflictos, de alguna u otra forma, presente en los horizontes políticos y sociales de todas las clases existentes del régimen capitalista. Por tanto, no es un instrumento monolítico del poder. Hecha esta aclaración, lo importante en cuanto a esta condición instrumental es, también, no explicar al derecho, a su utilización en los conflictos de clase, desde una perspectiva coyuntural sino desde una perspectiva de la larga duración, lo cual haré en uno de los apartados siguientes.

El que el derecho no sea el único instrumento de poder del régimen capitalista implica, por otro lado, la necesidad de explicar su papel en relación con los otros circuitos a través de los cuales se produce y reproduce el capitalismo (THOMPSON, 2001, p. 474). En primer lugar, nos encontramos con la necesidad de explicar la relación del derecho con las relaciones sociales de producción, esto eso, con la faceta o la cara

\footnotetext{
${ }^{4}$ Precisamente esta posibilidad de universalizar los intereses particulares de una clase constituye la característica distintiva de la instrumentalización del derecho moderno por parte del régimen capitalista (MARX \& ENGELS, 1974, pp. 71-72).
} 
económica de la reproducción capitalista. En este sentido, resulta necesario explicar si existe, históricamente y en cada sociedad determinada, una relación de complementariedad o de contrariedad entre la forma en que se producen, se distribuyen y circulan las mercancías. Una relación complementaria implica que, a pesar de que muchas veces parezca existir una contradicción entre el modelo de producción y la regulación jurídica de sus relaciones, en el largo plazo el derecho protege y fomenta la reproducción de las relaciones sociales que posibilitan las condiciones necesarias del modo de producción capitalista.

Para ello deberíamos observar no solamente el discurso de los derecho sociales, cuya perspectiva de distribución de la riqueza podría oponerse, aparentemente, a la apropiación privada y cada vez más concentrada del plusvalor y, por tanto, del trabajo social. Habría, más bien, que observar, históricamente situadas, las normas jurídicas que regulan tanto las relaciones de trabajo -si ayudan a mantener la relación de intercambio desigual entre patrón (capitalista) y trabajador, y en qué medida lo hacen de cara al nivel de conflictividad social—; también analizar aquellas normas que regulan la propiedad principalmente de los medios de producción -por ejemplo, si se fomenta o no la propiedad colectiva o común frente a la privada, la manera en que se regula la propiedad rural o la forma en que norma el papel de los bienes comunes, naturales, frente a las necesidades productivistas.

La explicación de todas estas cuestiones constituye una gran ayuda al momento de entender el por qué y el cómo pueden existir en nuestras sociedades, por un lado, un discurso de los derechos humanos cada vez más incluye y tendencialmente igualitario; por el otro, un conjunto de relaciones sociales, entre ellas buenas parte de la aplicación e interpretación de las normas jurídicas, cada vez más desiguales y excluyentes.

Igualmente resulta una explicación necesaria para comprender el papel del derecho en la conformación y reproducción de las relaciones sociales, analizar su relación con otros dos campos de lo social: lo político y lo cultural. En cuanto a lo primero, comprender que el derecho siempre es político, no en el sentido de ser producto de un régimen de gobierno o del estado, sino en cuanto es producto de relaciones sociales en las cuales lo político, más allá de lo partidista, siempre está en juego (ECHEVERRÍA, 2010: 77-78). De esta relación entre lo político y el derecho se puede comprender también, desde una perspectiva crítica, la relación entre el derecho, 
la democracia realmente existente, es decir, la ejercida a través de los aparatos burocráticos, y aquélla presente en ciertas prácticas sociales de las clases subalternas, la cual, muchas veces, entra en contradicción y antagonismo con la primera.

En cuanto a la relación con la cultura, resulta importante comprender en qué medida y de qué forma el derecho, su comprensión, su interpretación, su producción y su aplicación en cada contexto social históricamente determinado, se encuentra enmarcada en una ideología concreta, determinada a su vez por las formas culturales dominantes realmente existentes. Para esto sería de gran utilidad un cruce entre los estudios culturales y la crítica jurídica. Sin duda, desde la perspectiva que ofrezco existen muchas otras esferas de lo social cuyo análisis sería oportuno para la explicación adecuada del papel del derecho en la conformación y reproducción de las relaciones sociales.

La intención de lo anterior es resaltar un par de cuestiones que me parecen importantes para comprender lo jurídico dentro del marco de la totalidad social. En primer término, que ni lo jurídico, ni lo económico, ni lo cultural, ni cualquier otra esfera de la vida social, existen, en la vida real, como fenómenos separados los unos de los otros. Pues, si bien, es posible, y necesario, distinguir analíticamente entre ellos, lo social constituye una relación compleja entre fenómenos que son a la vez, y de manera indisociable, jurídicos, políticos, culturales y económicos (BRAUDEL, 1999: 9-11; THOMPSON, 2001: 474). De esta manera, la explicación crítica de lo jurídico requiere, de entrada, una apertura a las aportaciones de otros campos de saberes disciplinares, necesarios para comprender a lo jurídico desde una perspectiva de la totalidad social.

Precisamente para estar en las condiciones adecuadas para plantearnos lo jurídico desde una perspectiva de la totalidad es que considero que es importante recurrir a la historia social, pues ofrece una perspectiva abierta a los cruces interdisciplinarios que permiten partir desde una perspectiva de la totalidad social. De esto podemos dar algunos ejemplos que pueden ser útiles para comprender lo jurídico desde la historia: en primer término tenemos aquellos autores que conciben a la historia como el estudio de las civilizaciones humanas en el tiempo, lo cual implica que deben explicar todos los aspectos de la vida de dichas civilizaciones (por ejemplo sus relaciones de producción, pero también su matriz cultural) (BLOCH, 2001, p. 139; HOBSBAWM, 1998: 166-172); además, tenemos otras concepciones que comprenden a 
las relaciones sociales como un todo complejo dentro del cual lo económico, lo político y lo jurídico no constituyen sino caras de un mismo fenómeno, de tal manera que la historia, sin adjetivos, tiene como objetivo explicar lo social tomando en consideración todos estos aspectos de la vida (BENOIT, 1978: 58-59).

En general podemos decir que hay todo un conjunto de autores que conciben a la historia como una disciplina cuyo saber debe abarcar, al menos tendencialmente y como parte de un trabajo de investigación grupal, la totalidad social, así como realizar explicaciones que partan de la comprensión de todos los aspectos y factores que construyen la vida social en su totalidad (VILAR, 1998: 23-30). Por esta razón es que elijo ayudarme de la historia social, al menos de su marco teórico, conceptual y metodológico para explicar críticamente el papel del derecho moderno en la conformación y reproducción del régimen capitalista.

Además de su apertura interdisciplinaria y su perspectiva de totalidad que busca explicar la vida social desde la comprensión del conjunto complejo que constituyen las relaciones sociales, me parece que la historia social puede contribuir al análisis de la crítica jurídica puesto que, al contrario de la teoría jurídica y de la vieja historia, parte del estudio de la vida material, de las relaciones sociales, y no de las ideas o de la ideología dominante en una sociedad determinada -recordando que la idea dominante de cómo es una sociedad constituye la imagen de la clase dominante interiorizada por las clases subalternas (MARX \& ENGELS, 1974, p. 51).

De tal manera, la historia social no es contraria a la metodología de la crítica jurídica, la cual parte de una concepción materialista de lo social, a partir de la cual explicar tanto la producción de la vida material como su relación con las contenidos de conciencia -con las ideologías - de las diferentes clases sociales. En este sentido no solamente es la posibilidad de partir de una perspectiva de la totalidad para explicar lo jurídico, sino también de una concepción materialista, lo cual hace adecuado la colaboración entre la crítica jurídica y la historia social.

Ahora faltaría explicar una segunda cuestión acerca de la idoneidad de la elección de la historia social para ayudar a las explicaciones críticas sobre lo jurídico: el por qué partir de un estudio histórico, un abordaje que toma en cuenta el tiempo pasado para realizar sus explicaciones. La pregunta fundamental en este sentido sería, ¿Por qué o cómo explicar desde el pasado relaciones sociales que se reproducen en el 
presente?, ¿de qué sirve, para comprender los fenómenos jurídicos actuales, el papel actual de lo jurídico en la reproducción de las relaciones sociales hoy en día, partir desde la comprensión del pasado?, y, en este sentido, ¿por qué optar por la historia social en lugar de retomar la sociología crítica que ha tenido un mayor desarrollo en los trabajos de la crítica jurídica? Las siguientes dos secciones están destinadas a atender estas preguntas.

\section{PASADO PRESENTE, FUTURO. ¿DE QUÉ SIRVE LA HISTORIA SOCIAL DEL DERECHO PARA LA TRANSFORMACIÓN?}

La historia social nos puede ofrecer una explicación racional de los procesos históricos desde una perspectiva de la totalidad, dentro de la cual el derecho sería considerado, y desde una concepción materialista, la cual, en lugar de realizar una historia de los grandes personajes y de los grandes eventos coyunturales, se pueda construir una explicación desde las relaciones sociales, desde la reproducción material de la vida, sin dejar de tomar en cuenta el papel que tienen los contenidos de conciencia, la ideología en ésta (BRAUDEL, 1999: 24-29; THOMPSON, 2001: 481-489).

Ahora bien, está forma de proceder de la historia social contrasta con la manera en que, por lo general se realiza la historia del derecho, al menos en tres aspectos importantes y entrelazados el uno con el otro. El primero es por su carácter materialista que antagoniza con la postura idealista desde la cual se construye la historia del derecho tradicional. Este idealismo esta macado por dos cuestiones principales: la centralidad que ocupan en sus explicaciones los textos normativos leídos sin tener en consideración el conjunto de relaciones sociales desde las cuales emergen, se interpretan y aplican. Desde esta perspectiva, son las letras y no factores de emergencia y de aplicación de los textos normativos los datos básicos desde los cuales se construye la historia del derecho, la cual, por esta causa, más que una historia del derecho es una historia lineal de los textos normativos.

Muy relacionado con lo anterior se encuentra una tendencia a percibir al derecho como algo que mantiene un progreso lineal, independiente de los retrocesos en los procesos de democratización, distribución de la riqueza social y la profundización de los mecanismos de exclusión que operan en las sociedades actuales y que tiene sus 
precedentes directos, precisamente, en la historia de cómo ha surgido, interpretado y aplicado el derecho de acuerdo con las relaciones de fuerzas de las que surgió. Por otro lado, los análisis tradicionales de la historia del derecho no toman en cuenta las especificidades de los distintos tiempos históricos, por ejemplo, no distinguen el tiempo de las coyunturas a la larga duración, mucho menos distinguen la diferencia en la percepción del tiempo derivada de los contextos socio-político de los distintos procesos históricos.

Por último, buena parte de la historia del derecho se concentra en los grandes eventos y en los datos de los grandes personajes que intervinieron en el desarrollo de los sistemas jurídicos, lo cual limita la capacidad explicativa de sus resultados. Si bien, no es de obviar la intervención de los individuos en los procesos históricos, resulta central explicar la historia del derecho no desde éstos sino desde las relaciones y las estructuras sociales desde las cuales se construyen y se reproducen los sistemas jurídicos. Es decir, comprender la historia del derecho desde sus relaciones con la vida social.

En este mismo sentido, la historia social del derecho se diferencia de la historia del derecho tradicional por su tendencia interdisciplinaria. Si la segunda se centra, casi exclusivamente en los fundamentos conceptuales y en los saberes de la disciplina jurídica, tomando, de forma secundaria e incidental, datos de otras disciplinas, sin que éstos modifiquen, transformen o enriquezcan sus explicaciones (FOUCAULT, 2005, p. 15). ${ }^{5}$ La historia social del derecho remarcaría su apertura a las aportaciones de los saberes provenientes de otras disciplinas, como la ciencia política, la economía y la sociología,

Como se puede observar la historia social nos ofrece explicar el pasado del derecho moderno-capitalista desde una perspectiva distinta a las cuales que usualmente se emplean para hacerlo. La importancia de esta perspectiva es que nos permite explicar el pasado del derecho como realmente -social y materialmente- se ha construido y no desde la perspectiva limitante de los contenidos textuales de los documentos normativos, ni tampoco desde la óptica no pasa de los grandes acontecimientos y los grandes personajes sin remisión a su contexto socio-político y a las relaciones de fuerza involucradas.

\footnotetext{
${ }^{5}$ Constituye, en cierta manera, una historia interna del derecho.
} 
En este sentido, la historia social del derecho permitiría una reconstrucción racional que permita explicar no solamente dando cuenta de los cambios textuales de los enunciados normativos o de los discursos oficiales dados de cara a los procesos de las transformaciones de los sistemas jurídicos, como por ejemplo, los procesos constituyentes. Sino que, principalmente, nos ofrece una explicación de la cara jurídica de las relaciones sociales dentro de su contexto cultural, político y económico, por ejemplo, la relación de las transformaciones jurídicas con las del régimen capitalista en general, con los modelos de acumulación, con la manera de legitimación de su poder, etcétera. Por tanto, nos permite percibir la importancia del derecho en la totalidad de la vida social y los efectos de ésta en el discurso del derecho y el jurídico.

Así, la explicación racional del pasado del derecho moderno-capitalista se enriquecería con las aportaciones de la historia social. Además de ello, estas mismas aportaciones implicarían la necesidad de replantearse la importancia que tiene la comprensión del pasado para el entendimiento del presente. Una de las posturas de la historia social consiste precisamente en que la historia crítica no solamente se conforma por un conjunto de saberes eruditos, pero inservibles para la comprensión del presente, que corresponden más al anticuario, datos sobre la simple cronología de los acontecimientos o sobre sucesos coyunturales sin relacionarlos con el desarrollo estructural de lo social. El historiador, desde esta perspectiva, tiene como función explicar el pasado para comprender el presente, realizar su labor con una sensibilidad y un compromiso con la vida de su tiempo (BLOCH, 2001: 155-157; HOBSBAWM, 1998: 31).

Por otro lado, la historia social también ha dado cuenta de la dificultad de distinguir con nitidez entre los hechos que constituyen lo que denominados "el pasado" y aquellos que serían parte del "presente". Sus representantes han utilizado esta dificultad tanto para resaltar la imposibilidad de excluir a la historia del interés de las disciplinas dedicas a las explicaciones del tiempo presente, como la sociología y la economía; así como destacar la importancia que los aportes de estas disciplinas pueden tener para el desarrollo de la historia social.

Sin duda, en cuanto al tema de la historia del derecho, contrastando con el grueso de los estudios históricos desde el derecho que se enfocan a periodos y épocas remotas, las cuales en muchos casos no son reconducidas a la realidad actual sino de manera muy indirecta y remota; incorporar los postulados de la historia social a las 
explicaciones sobre el pasado del derecho ayudaría a comprender la manera en qué este forma parte de las opciones y las condiciones sociales y jurídicas del presente (BLOCH, 2001: 148-155; BRAUDEL, 1999: 78-82; HOBSBAWM, 1998: 217-218).

En este sentido, me parece que la historia social del derecho contribuiría no solamente a un mejoramiento cuantitativo del papel del derecho en la reproducción de las relaciones sociales, sino que, sobre todo, a una transformación cualitativa de la manera en que ésta se construye, tanto para los juristas como para aquellas personas que, desde otros campos disciplinares, se relacionan con el tema de lo jurídico. En todo caso, sus aportaciones podrían transforma, al cambiar la manera en que se percibe el papel del derecho en la totalidad social, la forma misma en que se construyen las ciencias sociales, pues pondrían a disposición de éstas una herramienta adecuada para explicar lo jurídico, aun cuando el derecho no sea su preocupación principal o lo sea de manera meramente incidental es importante contar con un método y una teoría adecuada para dar cuenta de lo jurídico -por ejemplo, al estudiar un proceso de ruptura social, muchas veces es inevitable hacer referencia a las transformaciones jurídicas, como la promulgación de nuevas constituciones.

Por último, si bien se reconoce que la historia, y específicamente la historia social, no tiene por objetivo, ni tampoco está en posibilidades, de ofrecer pronósticos certeros acerca de los acontecimientos futuros -el interés y la utilidad de la historia no se encuentra en su capacidad predictiva sobre el futuro, sino en su capacidad explicativa acerca del pasado-; también debe de tomarse en consideración que, a pesar de esto, la comprensión histórica puede aportar, a partir de una explicación adecuada del pasado, las mejores herramientas posibles para pensar el futuro, pues ayuda a conocer las condiciones sociales de emergencia y de transformación de las civilizaciones. En este sentido, debe recalcarse que el objetivo principal de la historia, de la historia social, consiste en la explicación de las transformaciones sociales, explicando los factores que intervienen en ella. Estos conocimientos en torno a la continuidad y la ruptura en los procesos históricos concretos pueden contribuir no solamente las condiciones de existencia presentes de las estructuras sociales, sino, que también puede resultar útil para reconocer las transformaciones sociales posibles en el futuro.

Ahora bien, un tema central en la historia social, que sería, desde mi punto de vista, necesario incorporar a la explicación histórica del derecho se encuentra en la 
distinción de las distintos tiempos históricos, específicamente, la distinción entre la larga duración y la corta duración. En cuanto al derecho esto representaría la capacidad de distinguir entre los factores y los efectos coyunturales de las transformaciones jurídicas, de aquellos que se encuentran relacionados de manera más estrecha con las estructuras sociales; específicamente sería útil para comprender, en la larga duración, la relación entre las transformaciones de los sistemas jurídicos y las del régimen capitalista, para observar hasta qué punto las primeras constituyen una de las condiciones necesarias para las segundas, es decir, hasta qué punto ambas son complementarias, o bien, en qué medida las primeras presentan horizontes de ruptura con el régimen capitalista. A esto estará destinada la sección siguiente del trabajo.

\section{ACONTECIMIENTO Y ESTRUCTURA. LAS DISTINTAS DURACIONES Y EL DERECHO MODERNO CAPITALISTA}

Como mencionamos al final del apartado anterior, uno de los aportes más importantes que nos puede brindar la historia social para la explicación del papel del derecho en la conformación y reproducción de las relaciones sociales se encuentra en la distinción de los diferentes tiempos históricos. Resulta oportuno comenzar por aclarar que los procesos históricos no están sujetos a un solo tipo de temporalidad -por ejemplo el tiempo biológico que medidos por horas, días, años y siglos, y que percibimos, muchas veces como único y universal-, sino que el tiempo para la historia está relacionado con el contexto social, político y económico vivido por cada proceso (Koselleck, 1993: 14-15).

De esta forma el tiempo histórico es distinto al biológico. El tiempo histórico, mejor dicho las temporalidades que constituyen uno de los objetos principales de la historia y desde las cuales realiza sus explicaciones, se relaciona más con los contextos socio-políticos de cada momento y de cada etapa que con la medición cronológica y la articulación simple y unilineal de los acontecimientos en ésta (BLOCH, 2001: 140-141). La explicación racional de la historia comienza desde la forma en que se ordenan los acontecimientos en temporalidades distintas a la biológica. Para nuestro trabajo las temporalidades que resultan fundamentales son la coyuntura, el tiempo corto, y la larga duración. 
Esta distinción principal entre la corta y la larga duración representa dos formas, por otro lado no antagónicas ni contradictorias totalmente entre sí, de comprender el tiempo histórico y explicar los distintos procesos. La primera se concentrará en explicar los hechos históricos desde la perspectiva del acontecimiento, que los explica desde sus factores de emergencia y efectos más inmediatos. La segunda los analiza desde su relación con la estructura social más perdurable, tratando de explicar los factores de su emergencia y sus efectos desde esta perspectiva (BRAUDEL, 1999: 29-30, 53-58, 64-66). ${ }^{6}$

En cuanto al análisis del derecho, esta distinción nos ofrece una veta muy importante para repensar la manera en que explicamos el papel del derecho en lo social. En primer término implica agregar un nivel de complejidad en su abordaje, al cual los estudios sobre el tema no están acostumbrados a emplear. Esto es así porque, tanto en la teoría como en la historia del derecho, se piensa al tiempo de manera uniforme y universal, sin distinguir entre aquellos efectos y causas que se presentan en el tiempo de la coyuntura y que modifican agresivamente el discurso del derecho, y aquellos que, pudiendo coincidir con los hechos históricos, por sus efectos y sus causas estructurales y en el largo plazo, sería más conveniente analizarlos desde la larga duración.

La larga duración incorpora a la historia crítica del derecho una herramienta esencial para comprender y explicar el papel del derecho y de sus transformaciones en la conformación de la estructura social de una civilización. Por un lado, permite analizar esta relación no solamente desde la perspectiva del acontecimiento, desde las transformaciones de lo jurídico pensadas desde la coyuntura, sino que permite pensarlas con relación a la estructura social que permanece en el tiempo. De manera que permitiría interpretar los cambios del discurso del derecho no solamente desde la forma en que se concibieron en el momento de su acontecimiento, sino desde los efectos que han tenido en la conformación de las relaciones sociales a lo largo de un periodo más extenso. Por ejemplo, si han modificado, y en qué condiciones, el modelo de acumulación, la forma en que se produce y en que circula la riqueza social.

Específicamente resulta importante explicar la relación de complementariedad, de ruptura o de transformación gradual que tiene un sistema jurídico en la larga

\footnotetext{
${ }^{6}$ Así Braudel distingue entre el tiempo corto del acontecimiento y la larga duración de las estructuras, ente la historia coyuntural, la episódica y la estructural, pues diferentes tiempos históricos requieren diferentes formas de explicarlos,
} 
duración con una estructura social, con un modo de producción y con un modelo de acumulación. Es decir, relacionar estructural y sistemáticamente el campo de lo jurídico, con el campo de lo político, lo económico y lo social. En este sentido, analizar el derecho no solamente desde una perspectiva de totalidad y desde una apertura a la interdisciplina, sino desde una mirada estructural, resulta esencial para comprender el papel que ha tenido en la conformación de las sociedades actuales y cómo sus transformaciones, más que simplemente incorporar nuevas normas jurídicas -con grados de efectividad diversos-, modifican las condiciones en que el régimen capitalista puede operar.

La larga duración puede ser útil en el análisis crítico del papel de los derechos sociales en las transformaciones del régimen capitalista. Para la teoría y la historia del derecho tradicionales, los derechos sociales representan el progreso constante del derecho hacia la igualdad no solamente formal sino material, de manera que se llegan a pensar como parte de un discurso que antagoniza y contradice a las prácticas sociales que se adscriben al poder salvaje del capitalismo . Además, como discurso que apela a la naturaleza humana universal, se piensan como ajenos a la ideología y a los conflictos de clase -los cuales solamente son relevantes para su emergencia, pero no para su interpretación y aplicación cotidiana (FERRAJOLI, 2000: 911-912).

Sin negar que los derechos sociales han representado mejorías en la vida material de las personas a las que han beneficiado, me parece que esta forma de explicarlos carece de un sustento histórico y, más bien, es posible mediante la generalización abstracta, al pensar a los derechos sociales como algo universal y no analizarlos desde los contextos sociales, políticos y económicos desde los cuales surgen y desde las relaciones de fuerza en medio de las cuales son ejercidos. En este sentido, pensamos que los derechos sociales, como cualquier parte del derecho, no pueden ser explicados sino a través de la consideración del contexto en el cual emergen, se interpretan y aplican. Lo anterior es un presupuesto para un análisis materialista del derecho.

En primer lugar, a pesar de la manera en que se presentan los derechos sociales y en general los derechos humanos-, a pesar de ser redactados en pactos internacionales y ser declarados universales, estos derechos -como cualquier derecho o norma jurídica - no surgen de la misma naturaleza de los seres humanos, sino que son 
producto relaciones sociales determinadas históricamente. Relaciones de fuerza dentro de cada país en el cual se constitucionalizan, así como también relaciones de fuerza mundiales cuando su reconocimiento proviene de pactos o tratados internacionales, los derechos sociales son siempre producto de algún tipo de conflicto social, la cual incluye no solamente el plano físico de la producción material de la vida, sino también el plano ideológico: los conflictos de clase son, siempre, en parte, una lucha ideológica. Los derechos sociales son siempre producto de las relaciones de fuerza entre las distintas clases sociales y de la mediación estatal-jurídica en sus conflictos, no son producto de un progreso humano, entendido como algo natural y colocado fuera de la política (SANDOVAL, 2013룰 157 y ss).

En este sentido, los derechos sociales no son nunca un discurso neutro, ni uno que permita una interpretación y una aplicación alejada de la política y de la economía, al contrario son siempre un terreno de disputa por la determinación de su sentido, son, desde esta perspectiva, un instrumento de clase, si bien, uno al cual cada una de las clases sociales tiene un acceso diferenciado. Esta disputa no termina con su inclusión en los textos constitucionales ni en los instrumentos internacionales, sino que se mantiene a lo largo de su interpretación cotidiana, pues sus alcances se interpretan de manera diferente de acuerdo a la posición de clase que se tenga: el derecho a la vivienda, a la salud, a la educación, al trabajo y al salario digno significan, día a día, cosas distintas para un empresario, para un trabajador y para una persona sin trabajo (ZAVALETA,1983: 55-6o). Lo único que ocurre es que el sentido oficial y dominante de dichos derechos se determina a través de la interpretación de los funcionarios estatales -predominantemente los órganos judiciales- y que dicha interpretación puede ser mantenida a través del uso de la violencia legítima que monopoliza, en nuestras sociedades, el discurso del derecho.

Por tanto, los derechos sociales se producen y se interpretan dentro de la lucha de clase. Ahora bien, la pregunta es, ¿qué ventajas explicativas puede ofrecer la consideración de la larga duración en la comprensión de la relación entre los derechos sociales y las transformaciones del régimen capitalista? La respuesta, desde la perspectiva de este trabajo y la investigación que lo motiva, sería la siguiente: permiten explicar a éstos no solamente a partir de los textos jurídicos en los cuales se incorporan al discurso del derecho -los cuales muchas veces se piensan haciendo abstracción del 
tiempo y del contexto en el cual se redactan y en el cual se aplican-, sino desde las relaciones sociales desde las cuales se producen y se interpretan. En otras palabras, permite relacionar el uso de los derechos sociales con la sociedad dentro de la cual emergen, observando su papel en las transformaciones de sus relaciones a lo largo de un periodo histórico.

En principio, los derechos sociales significan cosas diferentes a lo largo del tiempo y de acuerdo a las relaciones sociales y sus transformaciones. Sin duda, los derechos sociales en México en la etapa inmediatamente anterior a la revolución tenían un contenido distinto al que tienen en la época actual, no solamente porque los textos jurídicos que los contienen puedan haber cambiado o no, sino por el conjunto de relaciones sociales desde las cuales se interpretan, por las relaciones de fuerza y el nivel de conflicto entre las distintas clases sociales. Explicar estas transformaciones requiere pensar esos derechos sociales desde la larga duración (SANDOVAL, 2013b: 15-44).

Por otro lado, la perspectiva de la larga duración permite pensar estas transformaciones desde otra perspectiva. Si comúnmente, al menos en aquellos países en que se ha dado lo que conocemos como estado social y ahora vivimos en un modelo neoliberal, se piensan los cambios en la codificación, interpretación y aplicación de los derechos sociales como un proceso de desnaturalización, de desviación de los funcionarios públicos, de los textos normativos originales -por ejemplo, en México, con relación a la Constitución Política de 1917. En este sentido, la limitación progresiva de los derechos sociales constituiría un error de comprensión de su sentido original por parte de quienes gobiernan, lo cual no afectaría ni estaría directamente relacionado con los derechos sociales en sí mismos.

La larga duración permite explicar las transformaciones de los derechos sociales, no solamente a partir de errores o de interpretación coyunturales, sino a partir de las relaciones sociales que le dan su sentido en cada etapa del proceso histórico y, principalmente, desde una perspectiva estructural, desde la relación de todas estas transformaciones con el proceso histórico general; sobre todo, estaría en condiciones de explicar estos cambios con relación a la transformaciones generales de la vida social, para explicar las causas estructurales -económicas, políticas, etcétera- de los cambios de los alcances y contenidos de los derechos sociales, así como también los efectos que 
éstos han tenido en la manera en que se produce la vida materia y se distribuye el producto social en cada civilización.

De esta manera, pensamos que la larga duración puede ser útil para interpretar a los denominados estados sociales desde una perspectiva distinta, la cual se pregunte no solamente por las condiciones sociales y los efectos de su declive a nivel nacional, regional y mundial, sino que, sobre todo, se cuestione y explique el papel que éstos han tenido en la conformación de los sistemas políticos y económicos actuales. En otras palabras, estaría en condiciones de explicar cuál es el papel del estado social en las transformaciones del régimen capitalista en cada uno de nuestros países y en nuestra región. Esta explicación es relevante no solamente para aquellos países en los cuales no existe más este estado social, sino desde la perspectiva de la coyuntura actual de nuestra región en la cual han emergido nuevos estados sociales.

\section{AMÉRICA LATINA Y EL DERECHO MODERNO CAPITALISTA. HACIA UNA HISTORIA SOCIAL REGIONAL DEL DERECHO}

A pesar de que es muy pronto para arribar a resultados concluyentes, puesto que el presente trabajo refleja los primeros pasos de una investigación en curso. A estas alturas es posible, y me parece pertinente, contar con una sección que explique, sino el tema de cómo y en qué condiciones se podría construir una historia social del derecho desde y para América Latina, sí las razones por las cuales pienso que esta tarea es importante y de utilidad para nuestra región.

En primer lugar, como bien sabemos, tres países, Venezuela en 1999, Ecuador en 2008 y Bolivia en 2009, han promulgado nuevas constituciones en la vuelta de siglo. Lo importante, aunque no es una cuestión menor, no lo encontramos en los texto constitucionales y en sus múltiples innovaciones (en la manera que plantean, o replantean el papel y la estructura del estado en la vida social, en la incorporación de concepciones de democracia distintas a la liberal y en la transformación de la jerarquía comúnmente aceptada de los derechos humanos y la incorporación de nuevos derechos -de la Naturaleza, la soberanía alimentaria, la autodeterminación, entre otros). ${ }^{7}$ Lo

\footnotetext{
${ }^{7}$ Todo lo cual puede ser consultado en la abundante bibliografía acerca de las nuevas constituciones, un ejemplo de ello en Albert Noguera Fernández, Los derechos sociales en las nuevas constituciones latinoamericana (Valencia: Tirant lo Blanch, 2010).
} 
fundamental reside en entender estas constituciones como parte de tres procesos políticos que abrieron, si bien de forma momentánea, tensa y contradictoria, horizontes de transformación en nuestra región y, al menos de inicio, rompieron con las políticas neoliberales en dichos países.

Ahora bien, ninguno de estos procesos está exento de contradicciones internas y de conflictos de clases. Por el contrario, una de las tareas más importantes en sus coyunturas actuales se encuentra, precisamente, en explicar hasta qué punto se han transformado las relaciones sociales de explotación impuestas por el régimen capitalista y en qué medida su modo de producción y de acumulación continúa vigente en dichos países. Lo anterior, no para demeritar la importancia de los acontecimientos hasta ahora, sino para estar en condiciones de comprender sus alcances y sus limitaciones, para poder entender su presente de manera integral y pensar su futuro. ${ }^{8}$

Además de estos tres casos de nuevas constituciones, el cuadro de la coyuntura actual regional nos presenta con países en los cuales se profundizan, cada día, las políticas neoliberales de despojo, como por ejemplo, me parece el caso de México, de Colombia y, todavía, de Chile. Casos de países en los cuales, si bien se presentaron procesos políticos de ruptura con el neoliberalismo -sobre todo desde los movimientos sociales - el estado se ha reconfigurado y el modelo de acumulación neoliberal parece presentarse de forma apenas transformada -como podría ser el caso de Argentina, Brasil y Perú-; un conjunto de países que permanecen en la zozobra permanente en la zona del Caribe -por ejemplo Haití- y en parte de Centroamérica. Por último, como siempre un caso aparte, la situación de Cuba, no exenta de contradicciones.

De parte de la investigación surgen dos preguntas, la primera: ¿es posible, en estas condiciones locales heterogéneas, producto de las particularidades propias de cada proceso histórico nacional, reconstruir o articular una historia social del derecho desde una perspectiva regional?; la segunda, ¿en la coyuntura actual en la que se encuentra nuestra región de qué sirve una historia social del derecho? Para ambas, las respuestas incipientes, por encontrarse en etapa de construcción, y apenas un par de hipótesis.

\footnotetext{
${ }^{8}$ Por ejemplo, para Bolivia y Ecuador, véase Acosta, Alberto, et.al., El correísmo al desnudo (Quito: Montecristi Vive, 2013); Gaya Makaran (coord.), Perfil de Bolivia (1940-2009) (México: UNAM-Centro de Investigaciones sobre América Latina y el Caribe, 2011).
} 
En cuanto a la primera pregunta, nuestra hipótesis es que sí, es posible hablar de una historia social del derecho desde una perspectiva regional, si bien aún hay que construirla desde investigaciones nacionales, e interdisciplinarias, articuladas en un plano más general, sin perder de vista las particularidades de cada proceso histórico. La razón por la cual pensamos esto se encuentra en el papel que nuestra región -y por tanto, los países que la integran - tienen en la división internacional del trabajo, por las condiciones desiguales y de explotación - de la naturaleza y de la fuerza de trabajo- en las que todos ellos se han integrado a la economía mundial. Si bien esta condiciones adquiere diferentes matices dependiendo de las maneras en que se han resuelto las contradicciones y los conflictos de clase en cada país, lo cierto es que esta condición regional aporta un mínimo de condiciones comunes para poder articular estos procesos en una perspectiva más general que no termine siendo una mera abstracción (ZAVALETA, 2009: 113-115; MARINI, 1989: 14-23; MELGARITO, 2014: 74-114).

En cuanto a la segunda pregunta, nuevamente es algo que aún se encuentra en construcción, pensamos que la historia social del derecho para y desde América Latina puede ser de utilidad, primero para explicar las relaciones entre las reformas de los textos legales y los cambios en la interpretación y aplicación de las normas jurídicas y las transformaciones en las condiciones de existencia del régimen capitalista en nuestra región, lo cual sin duda es útil para comprender nuestras propias condiciones actuales de existencia. En segundo lugar, para poder analizar las perspectivas a futuro de las relaciones de fuerza y de los procesos políticos en marcha, reconociendo cuál ha sido la relación, en la larga duración, entre el derecho moderno y el sistema capitalista, cuál ha sido el papel del primero en la posibilización de la privatización de la acumulación y la explotación características del segundo. Esto nos sirve para evaluar las opciones que tenemos en el presente para construir un mejor futuro, si bien no es posible predecir las cosas por medio de este reconocimiento.

\section{CONCLUSIONES}

La historia social puede aportar herramientas de análisis muy importantes para la comprensión del papel del derecho en las transformaciones de las condiciones de existencia del capitalismo en nuestra región. No solamente en torno a una explicación 
más adecuada e integral de cómo ha existido en el pasado el derecho en nuestra región y de qué forma ha contribuido a las transformaciones del régimen capitalista en nuestros distintos países, sino, principalmente, para analizar cuál es y de dónde viene el derecho realmente existente en América Latina. Por otro lado, también resulta útil para la comprensión de las posibilidades del futuro del desarrollo de nuestra región.

Por estas razones, la construcción de una historia crítica del derecho resulta una tarea pertinente para los juristas, pero también para otros cientistas sociales, comprometidos con el pensamiento crítico. El presente trabajo, más que presentar conclusiones certeras, como serían propias de una investigación en su fase final, coloca las razones y las posibilidades de construcción de una disciplina aún inexistente en nuestra región.

\title{
SOCIAL HISTORY OF LAW FOR AND FROM LATIN-AMERICA
}

\begin{abstract}
The essay's objective is to propose the incorporation of the concepts of social history for the construction of a critical history of Law in Latin-America. In the first part the initial definition of Law and History is established, then the possibilities that social history can bring to the explanation of Law are argued; after that historical time is analyzed, especially, the concept of long duration in relation to Law; in the last part, some brief notes for the construction of a social history of law in Latin-America are advanced. Results are preliminary, because the investigation is on its first stages.
\end{abstract}

Keywords: Law; History; Social and economical Rights; Latin-America; México.

\section{BIBLIOGRAFÍA}

ACOSTA, Alberto et.al.2013. El correísmo al desnudo. Quito: Montecristi Vive, 2013.

BENJAMIN, Walter. (2007). Para una crítica de la violencia. en Walter Benjamin, Conceptos de Filosofía de la Historia, La Plata, Terramar, 2007.

BENOIT, Joachim. Hacer la historia social de Latinoamérica. Revista Dialéctica, vol. III, n. 5, pp. 9-22, 1978.

BLOCH, Marc. Apología para la historia o el oficio del historiador, México: Fondo de Cultura Económica, 2001. 
BRAUDEL, Fernand. La historia y las ciencias sociales. Madrid: Alianza, 1999.

CORREAS, Oscar. Crítica de la ideología jurídica. Ensayo sociosemiológico. México: Universidad Nacional Autónoma de México-Centro de Investigaciones Interdisciplinarias en Ciencias y Humanidades/Fontamara, 2005.

CORREAS, Oscar. Teoría del derecho. México: Fontamara, 2004.

ECHEVERRÍA, Bolívar. Valor de uso y utopía. México: Siglo XXI, 2010.

FERRAJOLI, Luigi. Derecho y Razón. Teoría del garantismo penal. Madrid: Trotta, 2000.

HART, Herbert L.A. El concepto de derecho. Buenos Aires: Abdeldo-Perrot, 1998.

HOBSBAWM, Eric J. Sobre la historia. Barcelona: Grijalbo Mondadori, 1998.

HOBSSBAWM, Eric J. El historiador entre la búsqueda de lo universal y la búsqueda de la identidad. Revista Historia social, n. 25, pp. 81-90, 1996.

KELSEN, Hans. Teoría pura del derecho. México: Porrúa, 2007.

KOSELLECK, Renihart. Futuro pasado: para una semántica de los tiempos históricos. Barcelona: Paidós, 1993.

MAKARAN, Gaya (coord.). Perfil de Bolivia (1940-20o9). México: UNAM-Centro de Investigaciones sobre América Latina y El Caribe, 2011.

MARINI, Ruy Mauro. Dialéctica de la dependencia. México: ERA, 1989.

MARX, Karl y Engels, Friedrich. La ideología alemana. México: Ediciones de Cultura Popular, 1974 .

MARX, Karl. El capital. Crítica a la economía política. Tomo 1. México: Fondo de Cultura Económica, 1999.

MELGARITO ROCHA, Blanca Estela. La criminalización de las protestas socioambientales en América Latina, una condición necesaria para la acumulación capitalista: el caso de México, Minera San Xavier; Argentina, Minera Bajo la Alumbrera. México: Universidad Nacional Autónoma de México, 2014.

NOGUERA FERNÁNDEZ, Albert. Los derechos sociales en las nuevas constituciones latinoamericanas. Valencia: Tirant lo Blanch, 2010.

SANDOVAL CERVANTES, Daniel. Derechos sociales y capitalismo en México y América Latina. Un acercamiento interdisciplinario desde la Crítica Jurídica. Revista 
Captura Criptica: direito, política, atualidade, Florianopolis, vol. 4, n. 1, pp. 157-185, $2013 a$.

Apuntes para una crítica de la epistemología del derecho contemporáneo. México: Universidad Nacional Autónoma de México, $2013 \mathrm{~b}$.

THOMPSON, Edward P. The essential E.P. Thompson. New York: The New Press, 2001.

VILAR, Pierre. Historia social y filosofía de la historia, Revista Dialéctica, México, vol. III, n. 5, pp. 147-164, 1978.

Pensar la historia. México: Instituto de Investigaciones "Dr. José María Luis Mora”, 1998.

WEBER, Max. Economía y sociedad. Esbozo de sociología comprensiva. México: Fondo de Cultura Económica, 2002.

WOLKMER, Antonio Carlos. História do Dereito no Brasil. Río de Janeiro: GenEditorial Forense, 2012.

ZAVALETA, René. Las formaciones aparentes en Marx. El capital. Teoría, estructura y método. Tomo 4. Coordinado por Pedro López Díaz, pp. 32-7o. México: UNAMDivisión de Estudios de Posgrado de la Facultad de Economía/Ediciones de cultura popular, 1983.

El estado en América Latina. La Paz: Los amigos del libro, 2009.

Trabalho enviado em 24 de novembro de 2014.

Aceito em 26 de janeiro de 2015. 